Pacific Journal of Mathematic 


\title{
COARSE UNIFORM CONVERGENCE SPACES
}

\author{
R. J. Gazik AND D. C. Kent
}

The coarse uniform convergence space is one which is the coarsest member of its convergence class. Each convergence space is compatible with a coarse uniform convergence structure. The regular topological spaces can be characterized as those whose coarse uniform convergence structures are uniformly regular or, equivalently, as those convergence spaces whose coarse uniform convergence structures are very strongly bounded. Every coarse uniform convergence space has a coarse completion. The coarse uniform convergence spaces which have uniformly regular completions are precisely the coarse uniform spaces.

o. Preliminaries. For any set $X$, let $F(X)$ be the set of all filters on $X$. The fixed ultrafilter generated by $\{x\}$ will be denoted by $\dot{x}$. (We shall hereafter use "u.f." as an abbreviation for "ultrafilter".) Let $\Delta$ be the diagonal in $X \times X$; we denote by $\dot{\Delta}$ the filter $\{A \subseteq X \times X: \Delta \subseteq A\}$.

DEFINITION 0.1. A uniform convergence structure $I$ on $X$ is a subset of $F(X \times X)$ which contains $\dot{\Delta}$, is closed under finite intersections, and satisfies the additional conditions;

(a) $\Phi \in I$ implies $\Phi^{-1} \in I$;

(b) $\Phi \in I$ and $\Phi \leqq \Psi$ implies $\Psi \in I$;

( c) If $\Phi, \Psi \in I$ and the composition $\Phi \circ \Psi$ exists, then $\Phi \circ \Psi \in I$.

We will use "u.c.s." as an abbreviation for "uniform convergence structure" or "uniform convergence space", where the latter term refers to a set equipped with a uniform convergence structure. The notion of a u.c.s. was introduced by Cook and Fischer [1], and a reader not already familiar with this notion can refer to [1] for background information.

A filter $\Phi \in F(X \times X)$ is defined to be $\Delta$-symmetric if $\Phi=\Phi^{-1}$

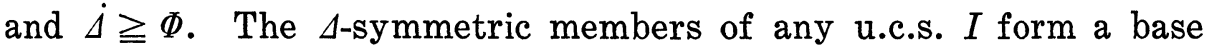
for $I$. A Cauchy filter for a u.c.s. $(X, I)$ is a filter $\mathscr{F} \in F(X)$ such that $\mathscr{F} \times \mathscr{F} \in I$. A u.c.s. is totally bounded if every u.f. is Cauchy.

DEFINITION 0.2. A convergence structure $q$ on a set $X$ is a function from $F(X)$ into the power set of $X$ satisfying the following conditions:

(a) $x \in q(\dot{x})$, all $x \in X$;

(b) $x \in q(\mathscr{F})$ and $\mathscr{F} \leqq \mathscr{G}$ implies $x \in q(\mathscr{G})$; 
(c) $x \in q(\mathscr{F})$ and $x \in q(\mathscr{G})$ implies $x \in q(\mathscr{F} \wedge \mathscr{G})$.

If $x \in q(\mathscr{F})$, then we write $\mathscr{F} \rightarrow x$ (read " $\mathscr{F}$ converges to $x$ "). If some u.f. finer than $\mathscr{F}$ converges to $x$, then $x$ is called on adherent point of $\mathscr{F}$.

Condition (c) of Definition 0.2 is often replaced by the weaker condition " $x \in q(\mathscr{F})$ implies $x \in q(\mathscr{F} \wedge \dot{x})$ " in defining the term "convergence structure"; our use of the stronger condition is motivated by the fact that Keller [2] has proved that each Hausdorff convergence structure (in the sense of Definition 0.2 ) is compatible with a u.c.s., and vice versa.

We will use the term space to mean convergence space, and abbreviate convergence structure by "c.s.". Throughout this paper we make the blanket assumption that all spaces are Hausdorff.

Given a u.c.s. $(X, I)$, the associated c.s. $q$ is obtained by designating $\{\Phi(\dot{x}): \Phi \in I\}$ to be the set of basic filters which $q$-converge to $x$. It should be noted that whenever $\mathscr{F} \rightarrow x, \Phi \in I$, and $\Phi(\mathscr{F})$ exists, then $\Phi(\mathscr{F}) \rightarrow x$; also note that $\Phi(\mathscr{F})$ always exists if $\Phi$ is $\Delta$ symmetric.

A u.c.s. is complete if every Cauchy filter converges. An excellent study of completions of u.c.s.'s can be found in [5].

A space is regular if $\mathrm{cl} \mathscr{F} \rightarrow x$ whenever $\mathscr{F} \rightarrow x$. Here, "cl" denotes the closure operator of the space; we use "cl" $\mathrm{l}^{n}$ " to denote the $n$-th interation of the closure operator. When used on the product $(X, q) \times(X, q)$, the closure operator is always assumed to be relative to the product c.s.. A u.c.s. $(X, I)$ is uniformly regular if $\mathrm{cl} \Phi \in I$ whenever $\Phi \in I$. It is clear that the c.s. associated with a uniformly regular u.c.s. is regular. More can be said if the u.c.s. is uniformly regular and totally bounded, but first some more definitions are needed.

Let $(X, q)$ be a space. The filter obtained by intersecting all filters which converge to $x$ is called the $q$-neighborhood filter at $x$, and denoted by $\mathscr{N}_{q}(x)$. If $\mathscr{N}_{q}(x) \rightarrow x$ for all $x$ in $X$, then $q$ is called a pretopology. The finest pretopology coarser than $q$ is denoted $\pi q$ and is characterized by: $\mathscr{N}_{q}(x)=\mathscr{N}_{\pi q}(x)$, for all $x \in X$. Note that $\pi q$ is a topology iff the closure operator for $q$ (or, equivalently, for $\pi q)$ is idempotent. A space $(X, q)$ is said to be almost topological if: (1) $q$ and $\pi q$ have the same convergence relative to u.f.'s; (2) $\pi q$ is a topology.

Proposition 0.3. If $(X, I)$ is a uniformly regular and totally bounded u.c.s. and $q$ the associated c.s., then $(X, q)$ is almost topological.

Proof. Let $\mathscr{F}$ be an u.f., $\mathscr{F} \geqq \mathscr{N}_{q}(x)$. Then $\dot{x} \geqq$ cl $\mathscr{F}$. Since $I$ is totally bounded, $\mathscr{F} \times \mathscr{F} \in I$; since $I$ is uniformly regular, $\operatorname{cl}(\mathscr{F} \times$ 
$\mathscr{F})=\mathrm{cl} \mathscr{F} \times \mathrm{cl} \mathscr{F} \in I . \quad$ But $\quad \dot{x} \geqq \mathrm{cl} \mathscr{F} \quad$ implies $\quad \mathrm{cl} \mathscr{F} \geqq(\mathrm{cl} \mathscr{F} \times$ cl $\mathscr{F})(\dot{x})$. So cl $\mathscr{F} \rightarrow x$, hence $\mathscr{F} \rightarrow x$. Thus $q$ and $\pi q$ have the same u.f. convergence.

Let $x \in \operatorname{cl}^{2} A$. Then there is an u.f. $\mathscr{F} \rightarrow x$ such that $\operatorname{cl} A \in \mathscr{F}$. By Lemma 2.1, [3], there is an u.f. $\mathscr{G}$ containing $A$ such that $\mathscr{F} \geqq \mathrm{cl} \mathscr{G}$. Note that $\dot{x} \geqq \mathrm{cl} \mathscr{F} \geqq \mathrm{cl}^{2} \mathscr{G}$. Thus, since $\mathrm{cl}^{2} \mathscr{G} \times \mathrm{cl}^{2} \mathscr{G}=$ $\mathrm{cl}^{2}(\mathscr{G} \times \mathscr{G}) \in I$, we have $\mathscr{G} \geqq\left(\mathrm{cl}^{2} \mathscr{G} \times \mathrm{cl}^{2} \mathscr{G}\right)(\dot{x})$, and so $\mathscr{G} \rightarrow x$. Thus $x \in \operatorname{cl} A$. This proves that $\pi q$ is a topology, and the proof is complete.

1. Coarse uniform convergence structures. Given a space $(X, q)$, let $[q]$ denote the set of all u.c.s.'s on $X$ compatible with $q$. It is well known (see [2] or [5]) that [q] contains a finest element $I^{q} ; I^{q}$ has a base consisting of filters of the form $\dot{\Delta} \wedge\left(\Lambda \mathscr{F}_{i} \times \mathscr{F}_{i}: i=\right.$ $1, \cdots, n\})$, where each $\mathscr{F}_{i} \rightarrow x_{i}$, and $\dot{x}_{i} \geqq \mathscr{F}_{i}$, for $i=1, \cdots, n$. Those u.c.s.'s which are finest elements of their respective convergence classes will be called fine u.c.s.'s. Note that a fine u.c.s. $I^{q}$ is always complete, and is uniformly regular whenever $q$ is regular. Similarly, $I$ will be called a coarse u.c.s. if $I \in[q]$ and $I$ is the coarsest u.c.s. in $[q]$. The coarse member of $[q]$ (if it exists) is denoted by $I_{q}$.

Proposition 1.1. For any space $(X, q), I_{q}$ exists and is generated by the collection $\mathscr{B}=\{\Phi \in F(X \times X): \Phi$ is $\Delta$-symmetric and $\Phi(\mathscr{F}) \rightarrow x$ whenever $\mathscr{F} \rightarrow x\}$.

Proof. It is easy to see that $\mathscr{B}$ is closed under finite compositions and finite intersections; thus $\mathscr{B}$ is the base for a u.c.s $J$ on $X$. From the construction of $\mathscr{B}$, it is clear that $J \in[q]$. If $I \in[q]$, $\Phi \in I$, and $\mathscr{F} \rightarrow x, \Phi \Delta$-symmetric, $\Phi(\mathscr{F}) \rightarrow x$; thus $\Phi \in J$, and it follows that $J=I_{q}$ is the coarsest member of $[q]$.

Proposition 1.2. If $(X, q)$ is a compact topological space and of the unique uniformity compatible with $(X, q)$, then

$$
I_{q}=\{\Phi \in F(X \times X): \Phi \geqq \mathscr{C}\} .
$$

Proof. Let $\mathscr{A}$ be an u.f. such that $\mathscr{A} \in I_{q}$. Since $X \times X$ is compact, $\mathscr{A}$ must converge, and by the Hausdorff property, \& converges to an element of the form $(x, x)$ in $X \times X$. Let $p_{1}, p_{2}$ be the two projection maps from $X \times X$ onto $X$. Then $\mathscr{A} \geqq p_{1} \mathscr{A} \times$ $p_{2} \mathscr{A} \geqq \mathscr{Y}$, since $\mathscr{V}$ is the filter of neighborhoods of $\Delta$. Since each member of $I_{q}$ is an intersection of u.f.'s in $I_{q}$, the result is established. 
If $q, p$ are convergence structures on $X$ and $q \geqq p$, then $I^{q} \geqq I^{p}$. The following example shows that the coarse u.c.s.'s are not so wellbehaved.

EXAMPLE 1.3. Let $(Y, r)$ be a completely regular topological space which is not locally compact, and let $(X, q)$ be a $T_{2}$ compactification of $(Y, r)$ such that $Y \subseteq X$. Let $p$ be the topology on $X$ such that the $p$-neighborhood filter $\mathscr{N}_{p}(y)$ at $y \in Y$ is generated by $\mathscr{N}_{r}(y)$ (considered as a filter base on $X$ ), and with $\mathscr{N}_{p}(x)=\dot{x}$ for $x \in X-Y$. Note that $q \leqq p$, and also that $I_{q}$ is generated by $\mathscr{Y}$, the unique uniformity for $(X, q)$. Let $\Delta_{X}$ be the diagonal in $X \times X$, and let $J$ be the u.c.s. on $X$ generated by $\left\{\Phi \wedge{\dot{\Delta_{X}}}_{X}: \Phi \in I_{r}\right\}$. If $I_{q} \leqq I_{p}$, then $I_{q} \leqq J$; in other words, $\mathscr{V} \leqq \Psi$ for all $\Psi \in J$. Consequently, $\left.\mathscr{Y}\right|_{Y \times Y} \leqq \Phi$, for all $\Phi \in I_{r}$. This implies that $(Y, r)$ has a coarsest compatible uniformity, contrary to the assumption that $(Y, r)$ is not locally compact. Thus $I_{q} \not I_{p}$.

Let $X$ be any set. For any finite cover $\mathscr{C}$ of $X$, let $H_{C}=$ $\bigcup\{A \times A: A \in \mathscr{C}\}$, and let $\Delta^{*}$ be the filter on $X \times X$ generated by $\left\{H_{C}: \mathscr{C}\right.$ a finite cover of $\left.X\right\}$. Reed, [6], defined a u.c.s. $(X, I)$ to be strongly bounded if $\Delta^{*} \in I$. If $\mathscr{F}$ is any u.f. on $X$, then $\mathscr{F} \times \mathscr{F} \geqq \Delta^{*}$; thus a strongly bounded space is totally bounded. Since $\Delta^{*}(\mathscr{G})=\mathscr{G}$ for all $\mathscr{G} \in F(X)$, the next result is an immediate consequence Proposition 1.1.

Proposition 1.4. Each coarse u.c.s. is strongly bounded.

We define a u.c.s. $(X, I)$ to be very strongly bounded if $\mathrm{cl} \Delta^{*} \in I$. Note that if $[q]$ contains any very strongly bounded member, then $I_{q}$ is very strongly bounded.

The next theorem gives several characterizations of a $T_{3}$ topological space in terms of its compatible coarse u.c.s..

THEOREM 1.5. The following statements about a space $(X, q)$ are equivalent:

(1) $(X, q)$ is a regular topological space;

(2) $I_{q}$ is uniformly regular;

(3) $I_{q}$ is very strongly bounded;

(4) There is a $\Delta$-symmetric filter $\Phi \in I_{q}$ such that, for each $A \subseteq X \times X, \operatorname{cl} A=\bigcap\{V \circ A \circ V: V \in \Phi\}$.

Proof. (2) implies (3). This follows immediately from Proposition 1.4. (3) implies (4). Let $\Phi=\mathrm{cl} \Delta^{*}$; by assumption, $\Phi \in I_{q}$. First note that for any u.f. $\mathscr{F}$ on $X, \mathscr{F} \times \mathscr{F} \geqq \Delta^{*}$. Thus, if $\mathscr{F} \rightarrow x$, 


$$
(\mathscr{F} \wedge \dot{x}) \times(\mathscr{F} \wedge \dot{x}) \geqq \mathrm{cl} \mathscr{F} \times \operatorname{cl} \mathscr{F} \geqq \mathrm{cl} \Delta^{*}=\Phi .
$$

Let $A \subseteq X \times X$, and let $(x, y) \in \operatorname{cl} A$. Then there is an u.f. $\mathscr{A} \rightarrow(x, y)$ such that $A \in \mathscr{A}$. By the observations of the preceding paragraph, $\dot{x} \times p_{1} \mathscr{A} \geqq \Phi$ and $p_{2} \mathscr{A} \times \dot{y} \geqq \Phi$. Given $V \in \Phi$, choose $W \in \mathscr{A}$ such that $\{x\} \times p_{1} W \cong V$ and $p_{2} W: \times\{y\} \subseteq V$. Since $W \cap A \neq \varnothing$, it follows immediately that $(x, y) \in V \circ A \circ V$. Thus,

$$
\operatorname{cl} A \subseteq \bigcap\{V \circ A \circ V: V \in \Phi\} .
$$

Conversely, let $(x, y) \in V \circ A \circ V$, all $V \in \Phi$. Choose $\left(a_{V}, b_{V}\right) \in A$, $\left(x, a_{V}\right) \in V$, and $\left(b_{V}, y\right) \in V$. The filter $\mathscr{A}$ on $X \times X$ generated by the net $\left(a_{V}, b_{V}\right)_{V \in \Phi}$ has the properties: $A \in \mathscr{A}$ and $\mathscr{A} \rightarrow(x, y)$. Thus $(x, y) \in \operatorname{cl} A$.

(4) implies (2). If $\Phi \in I_{q}$ is as indicated in (4) and $\Psi \in I_{q}$, then $\operatorname{cl} \Psi \geqq \Phi \circ \Psi \circ \Phi$, which implies that $\operatorname{cl} \Psi \in I_{q}$.

(2) implies (1). By Proposition 0.3, $I_{q}$ is almost topological. Thus, cl $\Delta^{*}$ has a filter base of sets of the form $\left\{H_{\mathscr{C}}: \mathscr{C}\right.$ a finite cover of $X$ by closed subsets of $X\}$. Since (2) and (3) are equivalent, $\operatorname{cl} \Delta^{*} \in I_{q}$. It is therefore sufficient to show that $\left(\mathrm{cl} \Delta^{*}\right)(\dot{x})$ is the $\pi q$-neighborhood filter at $x$, for all $x \in X$. This will establish that $q$ is a topological space; that $(X, q)$ is regular follows from the fact that $I_{q}$ is uniformly regular.

Let $B=\bigcup\left\{A_{i} \times A_{i}: i=1, \cdots, n\right\}$ be a basic member of $\mathrm{cl} \Delta^{*}$, where $A_{1}, \cdots, A_{n}$ are closed. Then $x \in A_{i}$ for some $i$, say $x \in A_{1}$. If $A_{1} \in \mathscr{N}_{\pi q}(x)$, we are done; otherwise, there is an u.f. $\mathscr{G} \rightarrow x$ such that $A_{1} \notin \mathscr{G}$. Thus some other $A_{j}$, say $A_{2}$, is in $\mathscr{G}$ and, since all of the $A_{i}$ 's are closed, $x \in A_{2}$. We can continue in this way until we either find an $A_{i} \in \mathscr{N}_{\pi q}(x)$, or else show that $x \in A_{i}$ for all $i$. Either way, it follows that $B(x) \in \mathscr{N}_{\pi q}(x)$.

(1) implies (3). By Proposition 1.1, it is sufficient to show that $\left(\mathrm{cl} \Delta^{*}\right)(\mathscr{F}) \rightarrow x$ whenever $\mathscr{F} \rightarrow x$. Given $\mathscr{F} \rightarrow x$, let $V$ be a closed neighborhood of $x$. Let $U$ be an open neighborhood of $x$ such that $U \subseteq V$. Choose $F \in \mathscr{F}$ such that $F \cong U$. Then

$$
B=(V \times V) \cup(\operatorname{Co} U \times \operatorname{Co} U) \in \operatorname{cl} \Delta^{*},
$$

and $B(F)=V . \quad$ This implies that $\left(\operatorname{cl} \Delta^{*}\right)(\mathscr{F}) \geqq \mathscr{N}_{q}(x)$, and the proof of Theorem 1.5 is complete.

2. Completions of coarse uniform convergence structures. From the study of uniform spaces, it is well known that locally compact topological spaces are the only ones which have a compatible "coarse uniformity", and that, when it exists, a coarse uniformity has a one-point completion which yields the one-point compactification 
of the space. In this section, we establish analogous results for coarse u.c.s.'s.

Starting with a space $(X, q)$, let $\mathscr{C}_{q}$ be the set of all Cauchy filters relative to $I_{q}$. Two Cauchy filters $\mathscr{F}, \mathscr{G}$ are said to be equivalent if $\mathscr{F} \wedge \mathscr{G}$ is a Cauchy filter (which is the same thing as saying $\mathscr{F} \times \mathscr{G} \in I_{q}$ ). Reed, [5], has shown that the set of Cauchy equivalence classes can be regarded as the underlying set for any completion of a u.c.s.. In particular, if all nonconvergent Cauchy filters of a u.c.s. $(X, I)$ are in the same equivalence class, then every completion of $(X, I)$ is necessarily a one-point completion.

In accordance with the convention adopted in the preliminary section, the term "completion" means Hausdorff completion.

Proposition 2.1. Let $(X, q)$ be a space. Then $\mathscr{F} \in \mathscr{C}_{q}$ iff $\mathscr{F}$ converges, or else $\mathscr{F}$ is nonconvergent and has no adherent points.

Proof. Assume that $\mathscr{F} \in \mathscr{C}_{q}$, and let $\mathscr{F}$ have an adherent point $x$. Then there is an u.f. $\mathscr{G}$ and that $\mathscr{G} \rightarrow x$ and $\mathscr{G} \geqq \mathscr{F}$. Since $(\mathscr{G} \wedge \dot{x}) \vee \mathscr{F} \neq \phi$, if follows from the properties of a Cauchy structure (see [2] or [5]) that $\mathscr{F} \wedge \dot{x} \in \mathscr{C}$, and so $\mathscr{F} \rightarrow x$.

Conversely, assume that $\mathscr{F}$ is a nonconvergent Cauchy filter with no adherent point. Let $\Psi_{\mathscr{F}}=\dot{\Delta} \wedge(\mathscr{F} \times \mathscr{F})$. If $\mathscr{G} \rightarrow x$, it is easy to see that $\Psi_{\mathscr{F}}(\mathscr{G})=\mathscr{G} \rightarrow x$, and so $\Psi_{\mathscr{G}} \in I_{q}$ by Proposition 1.1. Thus, $\mathscr{F} \in \mathscr{C}_{q}$.

Proposition 2.2. For any space $(X, q)$, there is exactly one equivalence class of nonconvergent $I_{q}$-Cauchy filters Consequently, each completion of $\left(X, I_{q}\right)$ is a one-point completion.

Proof. Let $\mathscr{F}, \mathscr{G}$ be nonconvergent filters without adherent points, and let $\Psi_{\mathscr{T}, \mathscr{G}}=\dot{\Delta} \wedge(\mathscr{F} \times \mathscr{G}) \wedge(\mathscr{G} \times \mathscr{F})$. If $\mathscr{H} \rightarrow x$, then it is easy to check that $\Psi_{\mathscr{F}, \mathscr{S}}(\mathscr{H})=\mathscr{H} \rightarrow x$. Thus, by Proposition 1.1, $\Psi_{\mathscr{F}, \mathscr{G}} \in I_{q}$. This proves that $\mathscr{F}$ and $\mathscr{G}$ are in the same Cauchy equivalence class.

Let $(X, q)$ be a noncompact convergence space, $a \notin X$, and let $Y=X \cup\{a\}$. Define $p$ to be the finest c.s. on $Y$ satisfying the following conditions:

(1) If $x \in X$, then $\mathscr{F} \rightarrow x$ in $(Y, p)$ iff $\left.\mathscr{F}\right|_{X} \rightarrow x$ in $(X, q)$;

(2) $\mathscr{F} \rightarrow a$ in $(Y, p)$ iff $\mathscr{F} \geqq \mathscr{G} \wedge \dot{a}$, where $\mathscr{G}$ contains $X$ and $\left.\mathscr{G}\right|_{X}$ has no adherent points relative to $(X, q)$.

Clearly, $(Y, p)$ is a Hausdorff one-point compactification of $(X, q)$.

THEOREM 2.3. Every noncomplete, coarse u.c.s. $\left(X, I_{q}\right)$ has a 
coarse one-point completion.

Proof. The space $(Y, p)$ constructed in the preceding paragraph is compact, and therefore $\left(Y, I_{q}\right)$ is complete. We will show that $\left(Y, I_{p}\right)$ is a completion of $\left(X, I_{q}\right)$.

Let $J_{q}=I_{p_{X \times X} \times X}$; clearly $J_{q} \geqq I_{q}$. Let $\Phi \in I_{q}$. To show that $\Phi \in J_{q}$, it is sufficient to show that $\Psi=\Phi \wedge \dot{\Delta}_{Y} \in I_{p}$. Let $\mathscr{F} \rightarrow x$ in $(Y, p)$. If $x \neq a$, then $\Psi(\mathscr{F})$ has exactly the same convergence properties in $(Y, p)$ as does $\Phi\left(\mathscr{F}_{1}\right)$ in $(X, q)$ (where $\left.\mathscr{F}_{1}=\left.\mathscr{F}\right|_{X}\right)$. Since $\Phi\left(\mathscr{F}_{1}\right) \rightarrow x$ in $(X, q), \Psi(\mathscr{F}) \rightarrow x$ in $(Y, p)$. If $x=a$, then $\mathscr{F}_{1}$ has no adherent point in $(X, q)$. $\Psi(\mathscr{F})$ could fail to converge to a only if $\left.\Psi(\mathscr{F})\right|_{X}$ had an adherent point relative to $(X, q)$. If this were the case, then there would be an u.f. $\mathscr{G}$ on $X$ such that $G \geqq \Phi\left(\mathscr{F}_{1}\right)$ and $\mathscr{G} \rightarrow x$ in $(X, q)$. But then, we would have $\mathscr{F}_{1} \times \mathscr{G} \geqq \Phi$, which would imply $\mathscr{F}_{1} \rightarrow x$ in $(X, q)$, a contradiction. Thus $\Psi(\mathscr{F}) \rightarrow a$ in $(Y, p)$, and $\Psi \in I_{p}$.

The concluding theorem shows that the coarse u.c.s.'s which have uniformly regular completions are precisely the coarse uniform spaces.

THEOREM 2.4. The following statements about a space $(X, q)$, are equivalent.

(1) $(X, q)$ is a locally compact regular topological space.

(2) $\left(X, I_{q}\right)$ has a uniformly regular completion.

(3) $I_{q}$ is a coarse uniformity.

Proof. (1) implies (3). Under the assumptions of (1), the compactification $(Y, p)$ constructed above is the usual topological onepoint compactification. From Proposition 1.2, it follows that $I_{p}$ is a uniformity. But it is shown in the proof of Theorem 2.3 that $\left(X, I_{q}\right)$ is a subspace of $\left(Y, I_{p}\right)$, as so $\left(X, I_{q}\right)$ is also a uniformity.

(3) implies (2). Obvious.

(2) implies (1). A uniformly regular completion of $\left(X, I_{q}\right)$ induces a regular completion of the Cauchy structure $\mathscr{C}_{q}$. By Theorem 3.4, [4], it follows that there is a uniformity $\mathscr{U} \in[q]$ such that $\mathscr{C} \leqq \Phi$ for all $\Phi \in I_{q}$. But then $(X, q)$ must be compatible with a coarse uniformity, which establishes that $(X, q)$ is locally compact and regular.

\section{REFERENCES}

1. C. H. Cook and H. R. Fischer, Uniform convergence structures, Math. Ann., 173 (1967), 290-306.

2. H. H. Keller, Die Limes-Uniformisierbarkeit der Limesraume, Math. Ann., 176 (1968), 334-341.

3. D. C. Kent and G. D. Richardson, The decomposition series of a convergence space, 
Czech. Math. J., 23 (1973), 437-446.

4. — Regular completions of Cauchy spaces, Pacific J. Math., 51 (1974), 483490.

5. E. E. Reed, Completions of uniform convergence spaces, Math. Ann., 194 (1971), 83-108.

6. —, Proximity convergence structures, Pacific J. Math., 42 (1973), 471-485.

Received April 30, 1975,

Arkansas State University

AND

Washington State UnIVERSity 


\section{PACIFIC JOURNAL OF MATHEMATICS}

\section{EDITORS}

RICHARD ARENS (Managing Editor) University of California

Los Angeles, California 90024

\section{R. A. BEAUMONT}

University of Washington Seattle, Washington 98105
J. DugundjI Department of Mathematics University of Southern Californı Los Angeles, California 90007

D. Gilbarg AND J. Milgram Stanford University Stanford, California 94305

\section{ASSOCIATE EDITORS}

E. F. BECKENBACH

B. H. NeUMANN

F. WOLF

K. YosHidA

\section{SUPPORTING INSTITUTIONS}

UNIVERSITY OF BRITISH COLUMBIA CALIFORNIA INSTITUTE OF TECHNOLOGY UNIVERSITY OF CALIFORNIA MONTANA STATE UNIVERSITY UNIVERSITY OF NEVADA NEW MEXICO STATE UNIVERSITY OREGON STATE UNIVERSITY UNIVERSITY OF OREGON OSAKA UNIVERSITY

\author{
UNIVERSITY OF SOUTHERN CALIFORNIA \\ STANFORD UNIVERSITY \\ UNIVERSITY OF TOKYO \\ UNIVERSITY OF UTAH \\ WASHINGTON STATE UNIVERSITY \\ UNIVERSITY OF WASHINGTON \\ AMERICAN MATHEMATICAL SOCIETY \\ NAVAL WEAPONS CENTER
}




\section{Pacific Journal of Mathematics}

\section{Vol. 61, No. $1 \quad$ November, 1975}

Jiří Adámek, V. Koubek and Věra Trnková, Sums of Boolean spaces represent every

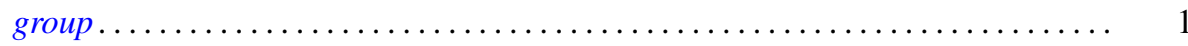

Richard Neal Ball, Full convex l-subgroups and the existence of $a^{*}$-closures of

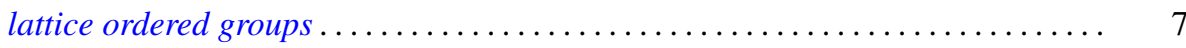



Gerald A. Beer, Starshaped sets and the Hausdorff metric . . . . . . . . . . . . . 21

Dennis Dale Berkey and Alan Cecil Lazer, Linear differential systems with



Harald Boehme, Glättungen von Abbildungen 3-dimensionaler

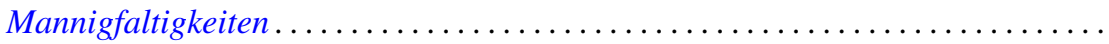

Stephen LaVern Campbell, Linear operators for which $T^{*} T$ and $T+T^{*}$

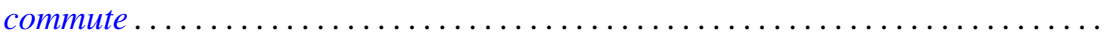

H. P. Dikshit and Arun Kumar, Absolute summability of Fourier series with

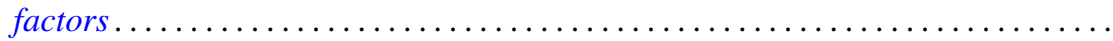

Andrew George Earnest and John Sollion Hsia, Spinor norms of local integral

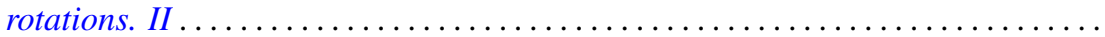

Erik Maurice Ellentuck, Semigroups, Horn sentences and isolic structures .........

Ingrid Fotino, Generalized convolution ring of arithmetic functions . . . . . . . . . . .

Michael Randy Gabel, Lower bounds on the stable range of polynomial rings .......

Fergus John Gaines, Kato-Taussky-Wielandt commutator relations and

characteristic curves

Theodore William Gamelin, The polynomial hulls of certain subsets of $C^{2}$

R. J. Gazik and Darrell Conley Kent, Coarse uniform convergence spaces. . .

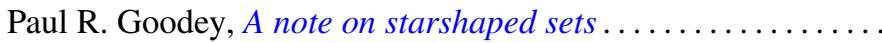

Eloise A. Hamann, On power-invariance

M. Jayachandran and M. Rajagopalan, Scattered compactification for $N \cup\{P\}$. . .

V. Karunakaran, Certain classes of regular univalent functions .

John Cronan Kieffer, A ratio limit theorem for a strongly subadditive set function in a locally compact amenable group .................

Siu Kwong Lo and Harald G. Niederreiter, Banach-Buck measure, density, and uniform distribution in rings of algebraic integers ........

Harold W. Martin, Contractibility of topological spaces onto metric spaces ....

Harold W. Martin, Local connectedness in developable spaces .

A. Meir and John W. Moon, Relations between packing and covering numbers of a tree.

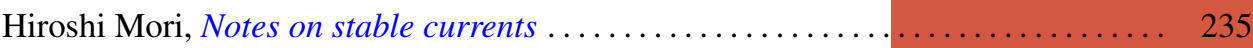

Donald J. Newman and I. J. Schoenberg, Splines and the logarithmic function . . . . 241

M. Ann Piech, Locality of the number of particles operator....

Fred Richman, The constructive theory of $K T$-modules .......

Gerard Sierksma, Carathéodory and Helly-numbers of

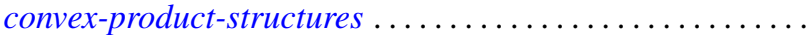

Raymond Earl Smithson, Subcontinuity for multifunctions .... . . 